\title{
A Robustness Simulation Method of Project Schedule based on the Monte Carlo Method
}

\author{
W. Na, P. Wuliang* and G. Hua \\ School of Economics and management, Shenyang Ligong University, Shenyang, 110159, China
}

\begin{abstract}
Since there are obvious uncertainties in task duration, the uncertainty will be passed step by step due to the precedence relationships among tasks. Therefore, one should pay attention to the robustness of project plan when scheduling the project. In this paper, the project plan robustness is evaluated as the probability of completing the project in the planning duration, the Monte Carlo method is used to simulate the practical implementation of the project plan considering the characteristics of task duration distribution, and thus a complete estimation approach for project plans is presented. This approach can help project managers to determine the project duration risk and identify the key tasks that influence the project plan robustness seriously at the beginning of project planning.
\end{abstract}

Keywords: Monte carlo method, project management, project robustness, simulation.

\section{INTRODUCTION}

Electricity Market participants always argue for better forecasts to maximize their profits. Forecasting electricity prices is important yet complex task because price series is non-stationary and highly volatile with non-constant mean variance. There are mainly two groups of forecasting methods consist of statistical method and artificial intelligence method [1-3]. A detailed review on price forecasting can be found in [4]. The invention presented in this paper relates to electricity price forecasting, and more particularly to price forecasting by employing error correction techniques.

Project planning and control is the core concern of project management. The project schedule is of great significance for project completion in due time, project economic performance, profit level and corporate reputation. The Critical Path Method (CPM) and Plan Evaluation Review Technology (PERT) are the two popular tools in project management. CPM and PERT are integrated in project practice to schedule project activities considering precedence relationships of activities and uncertainty. However, in most practice projects, a project schedule commonly consists of a large number of activities. Due to many precedence relationships among activities, the project schedule becomes a very complex project network. In this case, even though project leaders consider the uncertainty of each activity by using PERT, there is not an accurate method for them to evaluate the uncertainty of the whole project schedule. Therefore, the project risk increases and the robustness of the project schedule decreases along with the increasing of the number of activities.

Robustness is defined as "the ability of a system to resist change without adapting its initial stable configuration" or "network resilience" in complex network theory and it is an important concept in modern control theory and system science [1]. Robust project management is fairly new approach to project management. The present state of knowledge in robust project scheduling is presented in Al-Fawzan and Haourai (2005) [2], Herroelen and Leus (2005) [3] and Van Vonder et al. (2005) [4]. A robust schedule, constructed on the stage of project planning (thus, before the project starts), is usually defined one which will not differ very much from the final schedule realized during the execution of the project. Generally, two approaches are considered. The first type of project schedule robustness is called "quality robustness" and refers to the stability of the project planned make-span, i.e. the completion date of the whole project. The second type of project schedule robustness, called "solution robustness", refers to the details of the schedule, i.e. the starting times of the activities. In Van de Vonder et al. (2005) it is shown that the two approaches are not necessarily convergent and trade-off between the two robustness approaches if analyzed by means of simulation experiments. In this paper, we take as a starting point the approach proposed in AlFawzan and Haouari (2005) and put is in question. Since more and more research focus on the solution robustness, many computation methods of solution robustness are formulated and intensively investigated [4-6]. Compared with solution robustness, the quality robustness is difficult to measure and lacks computation formulation.

In this paper, we investigate the quality robustness of project schedule generated by CPM/PERT. Regarding the completion probability as the evaluation criterion of project robustness, the Monte Carlo Method is used to simulate the project execution and compute the completion probability. By implementing the method on Crystal Ball, a new method of evaluating the quality robustness of project schedule is realized. 


\section{MONTE CARLO METHODS USED IN PROJECT MANAGEMENT}

Monte Carlo methods (or Monte Carlo experiments) are a broad class of computational algorithms that rely on repeated random sampling to obtain numerical results; typically one runs simulations many times over to obtain the distribution of an unknown probabilistic entity. The name comes from the resemblance of the technique to the act of playing and recording results in a real gambling casino. They are often used in physical and mathematical problems and are most useful when it is difficult or impossible to obtain a closedform expression, or infeasible to apply a deterministic algorithm. Monte Carlo methods are mainly used in three distinct problem classes: optimization, numerical integration and generation of draws from a probability distribution [7]. Now, the tools and technologies for project scheduling is considerably popular and widely be acceptable. These lay a foundation for applying related theories in project management, and advance the promotion and application of Monte Carlo method in project management. The basic steps of Monte Carlo method using in project management is as follows:

Step 1: Investigate the basic project management problems and find out the core of it to determine the objective, and then formulate the objective and its relative risk parameters variable in a formulated mathematical model.

Step 2: Based on the risk identification and analysis of risk variables, determine their probability density function of each risk variable.

Step 3: According to precision requirements, set relevant properties such as the times of simulations. Randomly select values from generated random numbers and input them into the mathematical model built in Step 1 and achieve a special value of the project objective as a sample of random events.

Step 4: Repeat Step 3 and achieve N project objectives. Commonly, the more the times of experiment, the closer the probability distribution is close to the practice.

Step 5: Give the statistical analysis of the $\mathrm{N}$ samples achieved in Step 4 to get the distribution curve, test the probability distribution, estimate of the mean and standard deviation.

\section{THE SIMULATION OF PROJECT DURATION GENERATED BY CRITICAL PATH METHOD}

For projects with sophisticated technologies and many tasks, such as large-scale engineering projects, new product development, preparation of production technology, estimating the project duration as well as identifying critical tasks is of great significance. Also known as critical path analysis, the critical path method (CPM) is a widely used technique for analyzing and managing task sequences in large projects. Based on calculating how long it takes to complete essential steps of a process and analyzing how those steps interrelate, CPM is a visual and mathematical technique that gives managers the ability to effectively plan, schedule, and evaluate their projects.

However, in practical project management, the task duration in a project is an uncertainty variable under the influence of various factors. No matter project leaders have rich

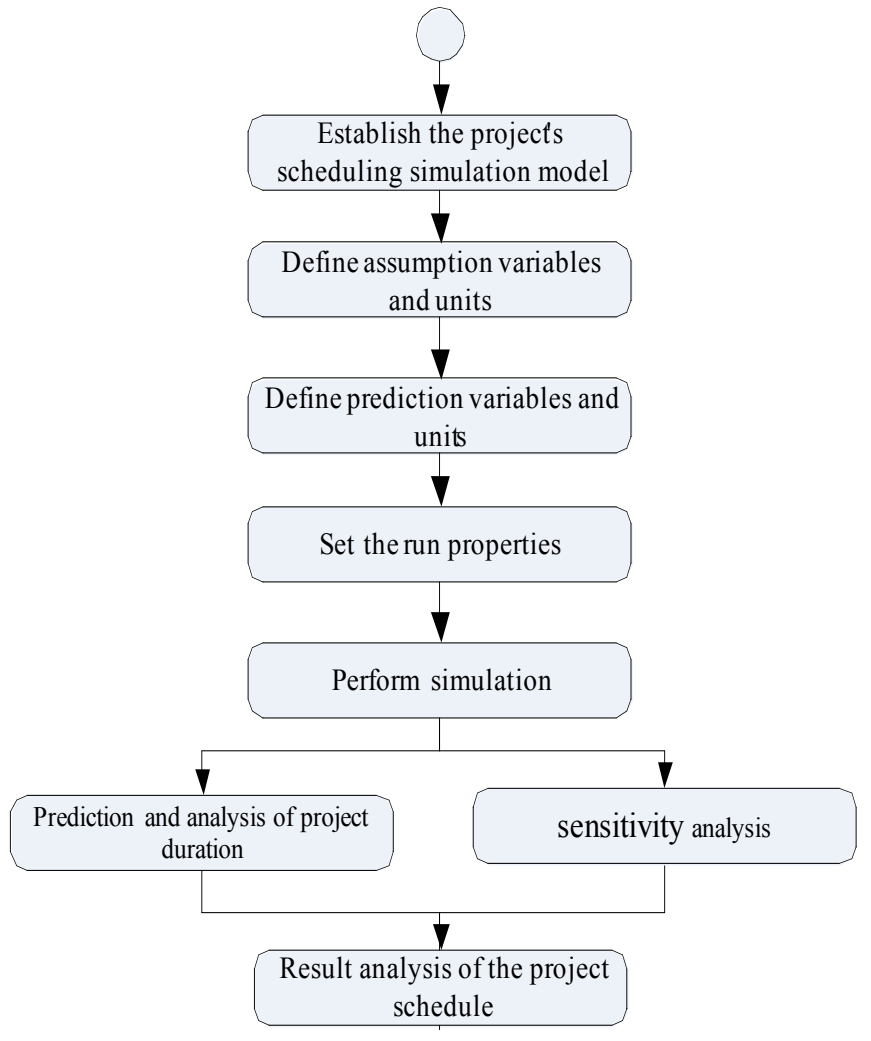

Fig. (1). The simulation of project scheduling based on Monte Carlo.

experiences and do their best to evaluate the task duration, there is always a considerable deviation between the planned duration and actual duration. That is, the uncertainty is the nature feature of project duration as well as task duration. This uncertainty is hard to be evaluated in the large-scale project management. Therefore, simulating the project duration by the Monte Carlo method can help the project leaders and other project stakeholders to evaluate the project duration and determine the robustness of the scheduled project plan. It will ease to quantify and control the project time risk.

In this paper, the Crystal Ball software, developed by Oracle, is used to implement the simulation of project scheduling based on Monte Carlo methods. The simulation process is shown as Fig. (1). A project case is used to illustrate the simulation process. The project activities and their precedence relationships are listed in Table. 1. It can be seen that there are 13 activities in the project, the precedence relationships, specialized in the fields "precedence" determine a project network for the project. The simulation procedure of Monte Carlo with Crystal Ball is described as follows:

(1) Establish the project's scheduling simulation model in Excel, which are the project list, in which all the activities, their precedence relationships as well as other properties such as duration, start time, and end time, as listed.

(2) Regard the unit cells residing activity durations as the assumption units and set a probability distribution to them. We assume that all the task durations are approximately subject to $\beta$ distribution. Complete the definition of assumption variables, including setting the maximum value as the 
Table 1. The project schedule generated by CPM.

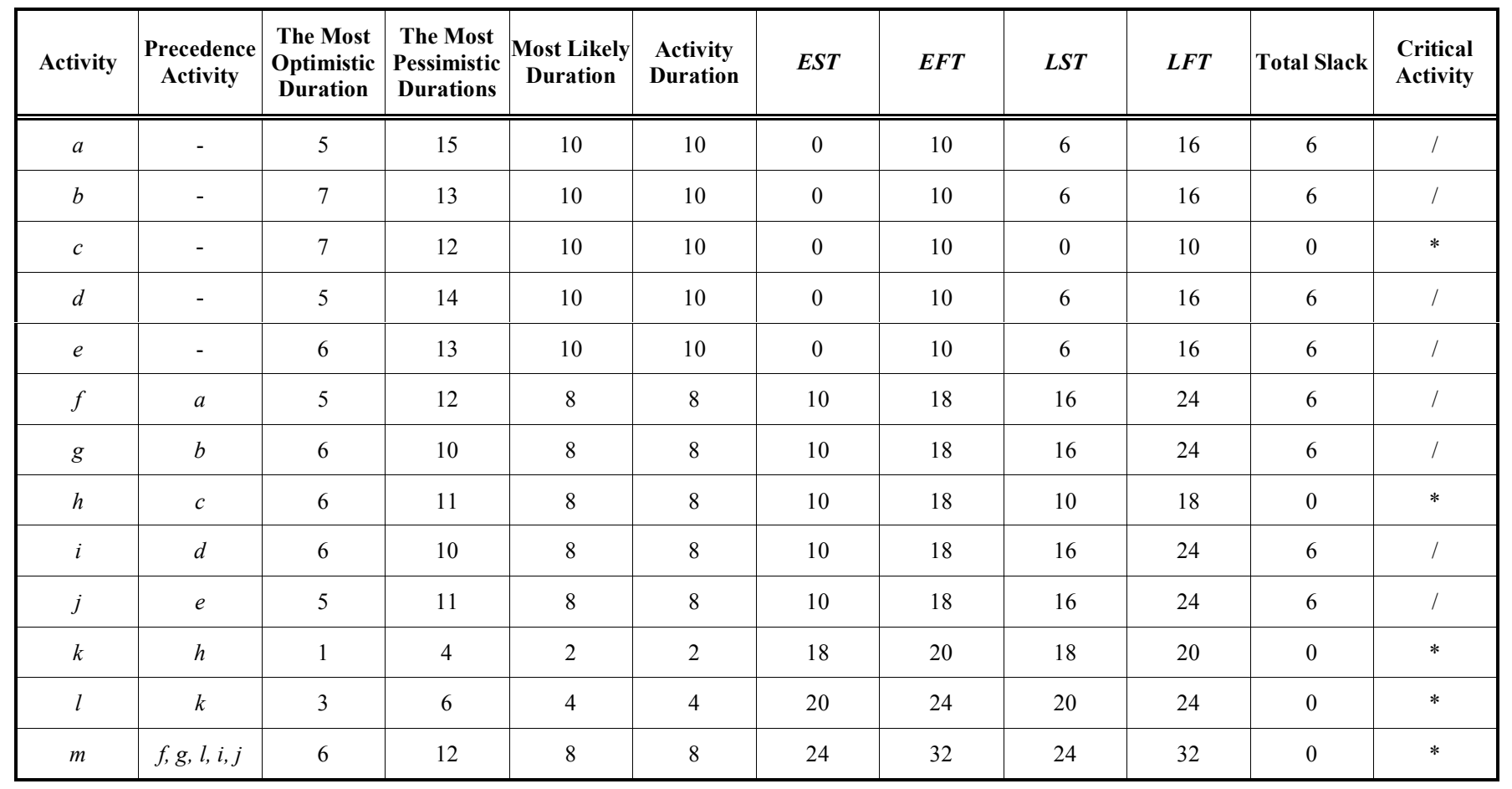

most pessimistic durations, the minimum value as the most optimistic durations.

(3) Regard the cell residing earliest finish time of the end activity as prediction unit, which is computed by the formulation in the mathematical model and varied with the assumption variables.

(4) Set the run properties and begin to perform simulation. Before the Monte Carlo simulation in project scheduling, the related parameters, such as the times of test, the types of sampling methods, the ways of improving simulation speed and accuracy control, should be set. In this paper, the number of test times is set 100, and the sampling method is Monte Carlo sampling method. After those parameters are set, the simulation process can be run.

(5) According to the project's actual requirements, analyze the related graphs and draw the further conclusion to help project leaders make the decision. For the project schedule, project leaders commonly focus on the completion rate and the effect of activities to the project schedule. Therefore, we will mainly analyze project duration prediction diagram as shown in Fig. (2) and sensitivity graph as shown in Fig. (3) generated by the simulation.

From project duration prediction diagram and the sensitivity graph, it can be seen that the minimum of project duration is 27.84 , and the maximum is 38.64 . In the model, the project duration is 32 days when the best possible durations are regarded as activity durations. From Fig. (2), it can be seen that the completion probability is $35.7 \%$, that is, the project robustness is $35.7 \%$. Project schedule completion rates reflect the risk level of the project implementation, and it is one of the important indicators to make decisions by project leaders. A project leader with conservative policy commonly tends to select the project schedule with higher completion rate.
Sensitivity analysis graph dynamically rendered each assume variable effects on predictor variables, indicating durations for each task in the project schedule impact on the duration of the project, as a percentage value reflects the influence of the size. Sensitivity analysis can clearly identify the project's critical tasks in project execution, focus and optimize these tasks, which can assure the stability and effectiveness of the entire program. In addition, the bar indicates that the hypothesis variable predictor variable is in the direction of positive impact or negative impact. From Fig. (3), it can be seen that activity $m$ affect project duration mostly, following by activity $h, c, k, l$, while the execution of activity $f$ has little effect on the whole project duration.

By CPM, the critical path of the project is $c \rightarrow h \rightarrow k \rightarrow l \rightarrow m$, which confirms to the result of the simulation approximately. Based on the levels of all the activities affecting project duration, project leaders can improve and optimize the project schedule.

The results of the Monte Carlo simulation of project scheduling can be used as the direct basis in the initial phase or the implementation. Therefore, project decision makers need organizing and saving related data and charts.

\section{CONCLUSION}

Planning and scheduling is the core content of project management. Although there is more research in the area of project scheduling and project planning scheduling correctly, the research on the project robustness is not mature. In this paper, we regarded project completion rate as the basis for measuring the quality and robustness of the project, presented an approach to simulate the CPM project schedule based on Monte Carlo methods. The approach is illustrated and verified by a practical project case. The approach allows 


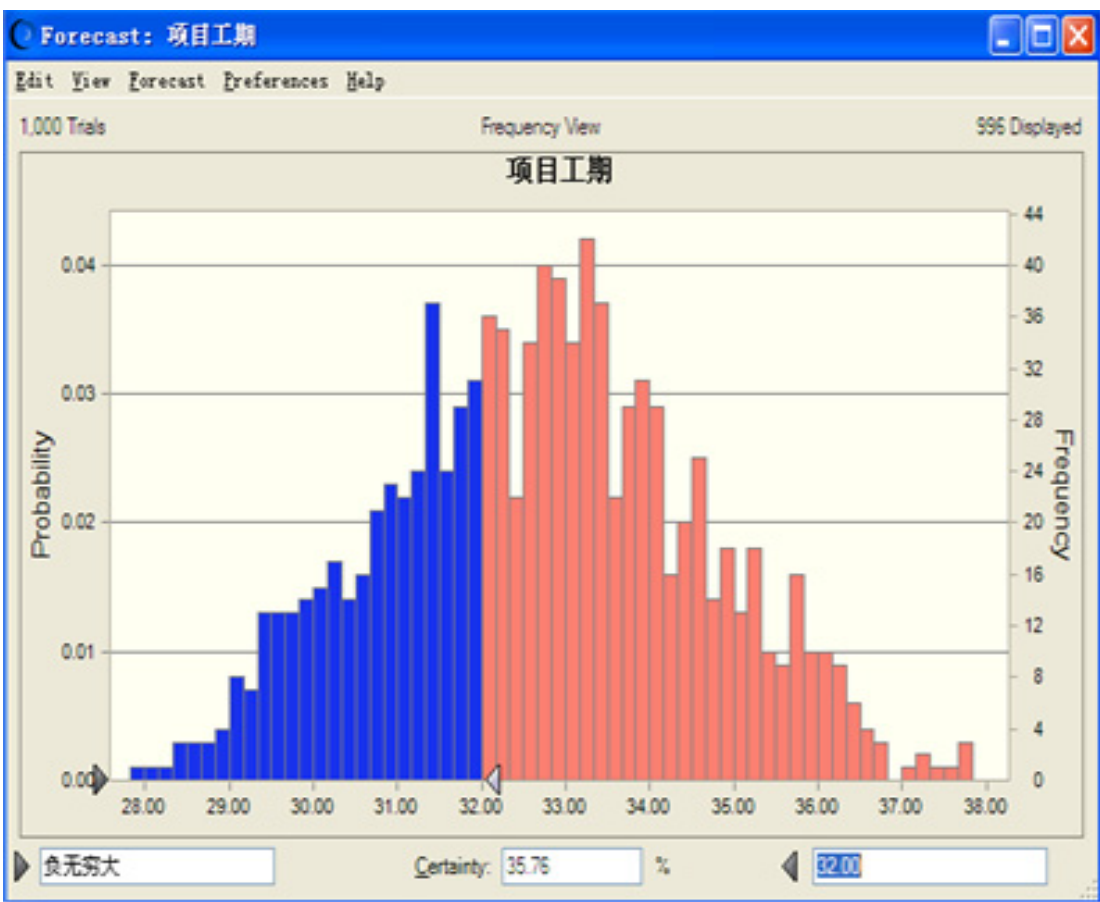

\begin{tabular}{|c|c|c|}
\hline & Statistic & Forecast values \\
\hline , & Trials & 1,000 \\
\hline & Base Case & 32.00 \\
\hline & Mean & 32.71 \\
\hline & Median & 32.73 \\
\hline & Mode & $\ldots$ \\
\hline & Standard Deviation & 1.89 \\
\hline & Variance & 3.56 \\
\hline & Skewness & 0.1152 \\
\hline & Kurtosis & 2.75 \\
\hline & Coeff. of Variability & 0.0577 \\
\hline & Minimum & 27.84 \\
\hline & Maximum & 38.64 \\
\hline & Mean Sod. Error & 0.06 \\
\hline
\end{tabular}

Fig. (2). Project duration prediction and statistics.

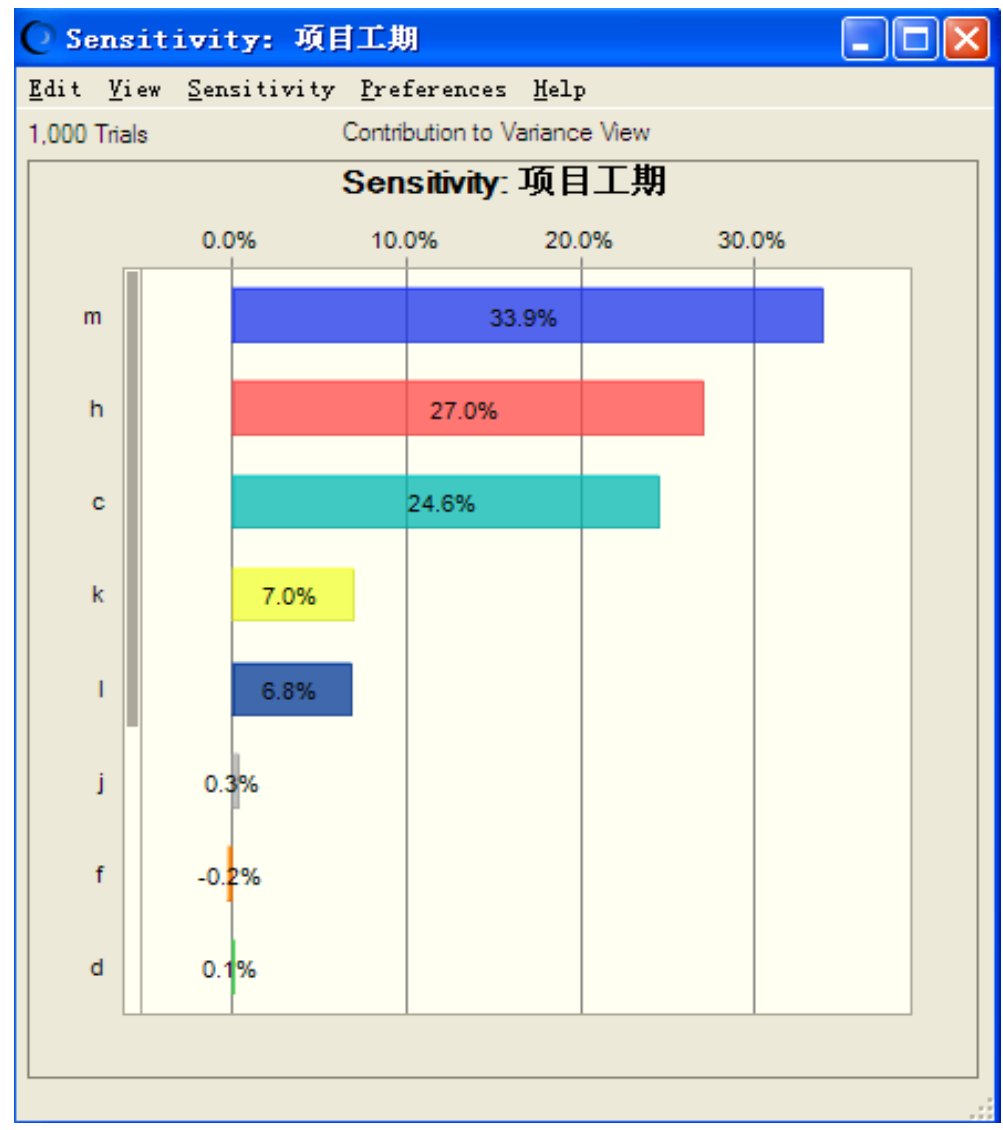

Fig. (3). Sensitivity analysis of project duration graph.

project leaders have a clear prospect to the project completion probability. During the process of project implementation, the approach can emphasize on the critical activities that affect the project duration seriously. This method allows project managers to project completion probability to have a clear sense of project risk. During the process of project implementation, the activities with great influence on the robustness of the project plan should be focused on to improve 
project management performance. The Monte Carlo Method is implemented by Crystal Ball Software with Excel, and the simulation process has good prospects due to its simplicity.

Further taking into account multiple resource constraints, implementing a specific project resource-supplying robust estimation method, is the direction for future study.

\section{CONFLICT OF INTEREST}

The authors confirm that this article content has no conflict of interest.

\section{ACKNOWLEDGEMENTS}

This work was supported by National Science Foundation of China under No. 71071100 and 71101097; the Liaoning BaiQianWan Talents Program Under No. 2013921033; the Human Resources Development Fundation of Shenyang under No. 2012141203022.

\section{REFERENCES}

[1] R. Albert, and A. L. Barabási. "Statistical mechanics of complex networks", Reviews of modern physics, vol. 74, pp. 47-57, January, 2002.

[2] M. A. Al-Fawzan, and M. Haouari. "A bi-objective model for robust resource-constrained project scheduling", International Journal of Production Economics, vol. 96, pp. 175-187, February, 2005.

[3] W. Herroelen, and R. Leus. "The construction of stable project baseline schedules", European Journal of Operational Research, vol. 156, pp. 550-565, March, 2004.

[4] S. Van de Vonder, E. Demeulemeester, W. Herroelen, and R. Leys, "The use of buffers in project management: The trade-off between stability and make-span", International Journal of Production Economics, vol. 97, pp. 227-240, February, 2005.

[5] M. A. Khemakhem, and H. Chtourou. "Efficient robustness measures for the resource-constrained project scheduling problem", International Journal of Industrial and Systems Engineering, vol. 14 pp. 245-267, February, 2013.

[6] J. Zhang, J. Liu, and R. Li. "Robustness-based discrete time/cost trade-off project scheduling". Computer Integrated Manufacturing Systems, vol. 11, pp. 2884-2892, November, 2013.

[7] F. Acebes, J. Pajares, J.M. Galán, and A. Lopez-Paredes "A new approach for project control under uncertainty. Going back to the basics". International Journal of Project Management, vol. 32, pp. 423-434, March, 2014.

Received: September 22, 2014

Revised: November 30, 2014

Accepted: December 02, 2014

(C) Na et al.; Licensee Bentham Open.

This is an open access article licensed under the terms of the Creative Commons Attribution Non-Commercial License (http://creativecommons.org/licenses/by-nc/3.0/) which permits unrestricted, non-commercial use, distribution and reproduction in any medium, provided the work is properly cited. 